\title{
Enhanced expression of the stemness- related factors OCT4, SOX15 and TWIST1 in ectopic endometrium of endometriosis patients
}

Katharina Proestling ${ }^{1}$, Peter Birner², Sukirthini Balendran², Nadine Nirtl', Erika Marton ${ }^{1}$, Gülen Yerlikaya ${ }^{3}$, Lorenz Kuessel ${ }^{1}$, Theresa Reischer ${ }^{1}$, Rene Wenzl ${ }^{1}$, Berthold Streubel ${ }^{2^{*}}$ (D) and Heinrich Husslein ${ }^{1}$

\begin{abstract}
Background: Current evidence suggests that endometrial-derived stem cells, spilled in the peritoneal cavity via retrograde menstruation, are key players in the establishment of endometriotic lesions. The aim of this study was to determine the presence and distribution of the stemness-related factors OCT4, SOX15, TWIST1 and DCAMLK1 in women with and without endometriosis.

Methods: Immunohistochemical analysis was used to determine stromal and epithelial expression of OCT4, SOX15, TWIST1 and DCAMLK1 in endometriosis patient (EP) endometrium $(n=69)$ and endometriotic tissue $(n=90)$ and in control endometrium $(n=50)$. Quantitative Real-Time PCR of OCT4, SOX15 TWIST1 and DCAMLK1 was performed in paired samples of EP endometrium and endometriotic tissue. Co-immunofluorescence staining was performed for OCT4 and SOX15. For statistical analyses we used unpaired t-test, Fisher combination test and Spearman test. For paired analyses, paired t-test and McNemar test were used.

Results: We detected a significant correlation between the expression of the established stem cell marker OCT4 and the stemness-related markers SOX15 $(p<0.001)$ and TWIST1 $(p=0.002)$ but not DCAMLK1. We showed a colocalization of SOX15 and OCT4 in epithelial and stromal cells of endometriotic tissue by coimmunofluorescence. A concordant expression of OCT4 and SOX15 in the same sample was observed in epithelial cells of the endometriotic tissue (71.7\%). The expression of stemness-related factors was not associated with proliferative or secretory phase of the menstrual cycle in endometriosis patients but was found to be differentially expressed during the menstrual cycle in the control group. Increased expression of epithelial OCT4, SOX15 and TWIST1 was detected in endometriotic tissue compared to EP endometrium in paired ( $p=0.021, p<0.001$ and $p<0.001)$ and unpaired analysis $(p=0.040, p<0.001$ and $p=0.001)$.
\end{abstract}

Conclusion: Our findings support the hypothesis that upregulation of stem cell-related factors contribute to the establishment of endometriotic lesions.

Trial registration: The study was approved by the institutional review board (545/2010 on 6th of May 2014) of the Medical University of Vienna (http://ethikkommission.meduniwien.ac.at/fileadmin/ethik/media/dokumente/register/ alle_2010.pdf).

Keywords: Endometriosis, Stem cells, OCT4, SOX15, TWIST1

\footnotetext{
* Correspondence: berthold.streubel@meduniwien.ac.at

${ }^{2}$ Department of Pathology, Medical University of Vienna, Waehringer Guertel

18-20, 1090 Vienna, Austria

Full list of author information is available at the end of the article
} International License (http://creativecommons.org/licenses/by/4.0/), which permits unrestricted use, distribution, and reproduction in any medium, provided you give appropriate credit to the original author(s) and the source, provide a link to the Creative Commons license, and indicate if changes were made. The Creative Commons Public Domain Dedication waiver (http://creativecommons.org/publicdomain/zero/1.0/) applies to the data made available in this article, unless otherwise stated. 


\section{Background}

Endometriosis is a benign gynecological disease, which is characterized by the presence of functional endometrial glands and stroma outside the uterine cavity [1]. The exact aetiology of endometriosis is unclear. One widely accepted hypothesis is that endometriosis originates from retrograde menstruation of endometrial cells which implant on peritoneal surfaces [2]. During the reproductive life of women the endometrium undergoes profound changes according to the different phases of the menstrual cycle [3]. Several studies revealed the presence of adult stem cells in the basalis layer as well as functionalis layer of the human endometrium [4-7]. These endometrium-derived stem cells, which are distributed by retrograde menstrual efflux, may contribute to the establishment of ectopic endometriotic lesions [7-11]. The monoclonal origin of some endometriotic lesions, long-time culture properties of cell clones established from endometriotic lesions and the isolation of progenitor cells from menstrual blood support this hypothesis [12-17].

Recent studies have been evaluating candidate markers for endometrial progenitor stem cells. Several general adult stem cell markers, such as bcl-2, c-kit (CD117), CD34 and noteworthy OCT4 have been identified in human endometrial tissue samples [4, 7, 18-22]. The transcription factor OCT4 is crucial for the maintenance of cell pluripotency and is known to be expressed in embryonic stem cells, germ cells and in adult stem cells [7, 23]. Furthermore, in vitro studies have shown that elevated expression of OCT4 and SOX2 in human endometrial cells contributed to reprogramming these cells into induced pluripotent stem cells (iPS cells) suggesting that OCT4 and SOX2 are stemness-related factors in human endometrium [24]. In endometriosis, the epithelial expression of OCT4 evaluated by immunohistochemistry was significantly higher in ectopic lesions compared to eutopic endometrium of patients with and without endometriosis. [25, 26]. Although the transcription factor OCT4 is a known key modulator for stem cell properties of primate stem cells, it further forms complexes with many different partners and displays various functions [27]. Several SOX-OCT transcription factor combinations have been identified and the cooperation correlates with the efficiency in producing iPS cells [28]. SOX15 for example strongly cooperates with OCT4 on the canonical pathway and has not been investigated in endometriosis so far [28].

The aim of this study was to analyze the role of stem cell-related markers in endometriosis. We correlated the expression of SOX15 and the potential stem cell factors TWIST1 (twist family bHLH transcription factor 1) and DCAMLK1 (doublecortin- and calmodulin kinase-like 1) with OCT4 expression. We analyzed the coexpression of SOX15 and OCT4 in epithelial and stromal cells of endometriotic lesions. Further we investigated the expression of stemness-related factors during the proliferative and secretory phase of the menstrual cycle, in patients with and without endometriosis and in different stages of endometriosis.

\section{Methods}

\section{Patients and tissue samples}

Tissue samples were obtained from 160 premenopausal women (mean age $34.5 \pm 6.4$ years), who underwent surgery at the General Hospital of Vienna between 2010 and 2014 due to the suspicion of endometriosis with or without infertility, chronic pelvic pain, benign adnexal masses, or uterine leiomyoma. The tissue samples have been collected in the context of a prospective cohort study called EMMA Study (EndoMetriosisMarkerAustria). The 160 cases consisted of 110 patients with endometriosis and 50 control patients who underwent laparoscopy and endometrial sampling with or without hysteroscopy due to benign adnexal masses, chronic pelvic pain or uterine fibroids. Exclusion criteria were pregnancy or breastfeeding less than 6 months prior to the beginning of the study, malignant disease, infectious diseases (HIV, hepatitis A, B and $\mathrm{C}$, tuberculosis) and autoimmune diseases. Among the 110 cases with endometriosis, we obtained matched samples of endometriotic and eutopic endometrium in 49 cases, exclusively endometriosis patient (EP) endometrium in 20 cases, and exclusively endometriotic tissue in 41 cases. The study was approved by the institutional review board (545/2010) of the Medical University of Vienna. All participants provided written informed consent. The matched sample tissues were collected during the same surgical procedure. Tissues were sliced in two. One part of the sample was snap-frozen in liquid nitrogen (LN2) immediately after surgical extraction and stored at $-80^{\circ}$ Celsius to minimize enzymatic degradation until final analysis. The other part of the tissue was fixed in $4 \%$ buffered formalin immediately after surgical extraction and stored overnight for processing of immunohistochemical analysis. Endometriosis was diagnosed histologically in all endometriosis patients. Staging was performed according to the revised American Fertility Society (rAFS) classification guidelines (I, $n=17$; II, $n=23$; III, $n=22$; IV, $n=25$ ) [29]. Cycle phase was determined by histopathological examination of endometrial samples by an experienced pathologist and was confirmed by date of last menstrual period.

Characteristics of the study populations are provided in Table 1.

\section{Quantitative Real-Time PCR (qRT-PCR)}

Briefly, total RNA was isolated from fresh frozen tissues with the Absolutely RNA miRNA Kit (Agilent, CA, USA) and reverse-transcribed with the SuperScript First-Strand 
Table 1 Description of the study population

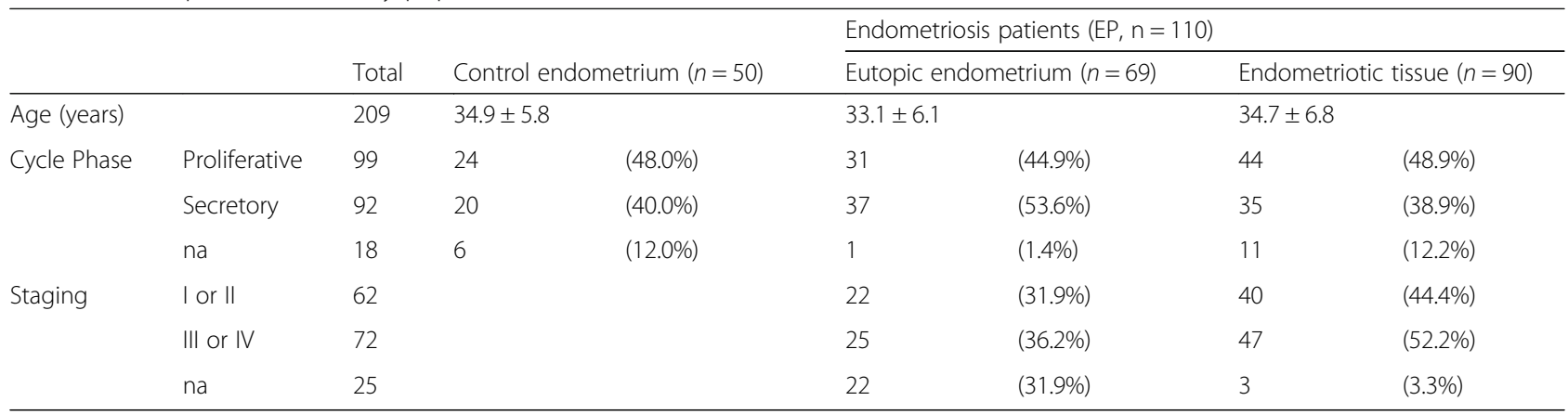

EP Endometriosis patient. Numbers of patients in each of the indicated subgroups are shown. Numbers in parentheses indicate the fraction of patients (\%) in each column in proliferative and secretory cycle phase or with low and high stage [28]. na, status not available

Kit (Invitrogen, CA, USA) according to the manufacturers' instructions. Each sample was analyzed by realtime PCR on an Applied Biosystems 7500 fast instrument, using gene-specific primers and fluorescent probes obtained from Applied Biosystems (CA, USA): OCT4, Hs00999632_g1; SOX15, Hs_00199511_m1; TWIST1, Hs_01675818_m1; DCAMLK1, Hs00178027_m1; GAPDH (control), Hs_99999905_m1, and ACTB (control), Hs_99999903_m1. The mRNA levels of OCT4, SOX15, TWIST1 and DCAMLK1 were normalized to those of ACTB and GAPDH in each sample by subtracting the mean $\mathrm{Ct}$ (threshold cycle) values of the controls from the $\mathrm{Ct}$ value of OCT4, SOX15,TWIST1 and DCAMLK1 as described previously [30]. For binary analysis, the cutoff was set at the median levels for SOX15 and TWIST1 expression.

\section{Immunohistochemistry (IHC)}

Immunohistochemical staining was performed on formalinfixed, paraffin-embedded tissues. Three-micrometer thick sections were cut and placed on glass slides. Heat antigen retrieval was performed in $10 \mathrm{mM}$ Sodium Citrate Buffer pH6. Nonspecific background staining was blocked by incubating in $\mathrm{H}_{2} \mathrm{O}_{2}$ and with Ultra $\mathrm{V}$ Block (Thermo Scientific, Ultra Vision LP Kit, TL-060-HL, MA, USA) according to the protocol. The following antibodies were used: the rabbit polyclonal anti-TWIST antibody (Abcam, ab50581, Cambridge, UK) was applied at a dilution of 1:1200 with Antibody Diluent with Background Reducing Components (Dako, S3022, Glostrup, Denmark), the mouse monoclonal anti-DCAMKL1 antibody (Abcam, ab88484, Cambridge, UK) at a dilution of 1:500, the rabbit monoclonal anti-OCT4 antibody (Abcam, ab109183, Cambridge, UK) at a dilution of 1:500, and the rabbit polyclonal anti-SOX15 (Abcam, ab55960, Cambridge, UK) at a dilution of 1:300. Ultra Vision LP Kit was used for detection according to the protocol (Thermo Scientific, Ultra Vision LP Kit, TL-060-HL, MA, USA). Finally, all slides were incubated with DAB-Substrate (Dako, K346811,
Glostrup, Denmark) and counterstained in Hematoxylin before they were dehydrated and mounted.

\section{Scoring and Immunohistochemical Analysis}

Prior to immunohistochemistry, endometriotic lesions consisting of well-defined glandular epithelial and stromal cells were identified in hematoxylin-eosin stained sections by a pathologist. Serial sections were cut from the chosen samples. A semiquantitative subjective scoring system to evaluate the localization, quantity and intensity of immunoreactivity was employed using light microscopy (200x magnification). In each sample, the staining for glandular epithelial cells and stromal cells was scored separately. The intensity of the staining was scored using a four-point scoring scale $(0$, negative staining; 1 , weak staining; 2 moderate staining, 3 , strong staining). The percentage of positively stained cells was again scored by a four-point scoring scale $(0$, negative staining; $1,1-35 \%$ positive cells; $2,36-70 \%$ positive cells; 3 , $>67 \%$ positive cells). The two scores were combined by multiplication to derive a final IHC score (0-9). For binary analysis, the cutoff was set at the median level of the final IHC score. Evaluations were performed in blind by two investigators. Positive (Seminoma) and negative (without primary antibody) controls were run concurrently. OCT4 (Fig. 1a, b) and SOX15 proteins (Fig. 1c, d) were expressed in the nucleus of the epithelial and the stromal cells of eutopic and ectopic endometrium. TWIST1 expression was observed in the cytoplasm and nucleus of epithelial and stromal cells (Fig. 1e, f). However, as a transcription factor, activated TWIST1 exerts its main function in the nucleus. Thus, for TWIST1, only nuclear staining of epithelial and stromal cells was evaluated. DCAMLK1 protein was expressed in the cytoplasm of the epithelial and stromal cells in eutopic and ectopic endometrium (Fig. 1g, h).

\section{Confocal Immunofluorescence Studies}

Immunofluorescence staining was performed on formalinfixed, paraffin-embedded tissue. Heat Antigen Retrieval 


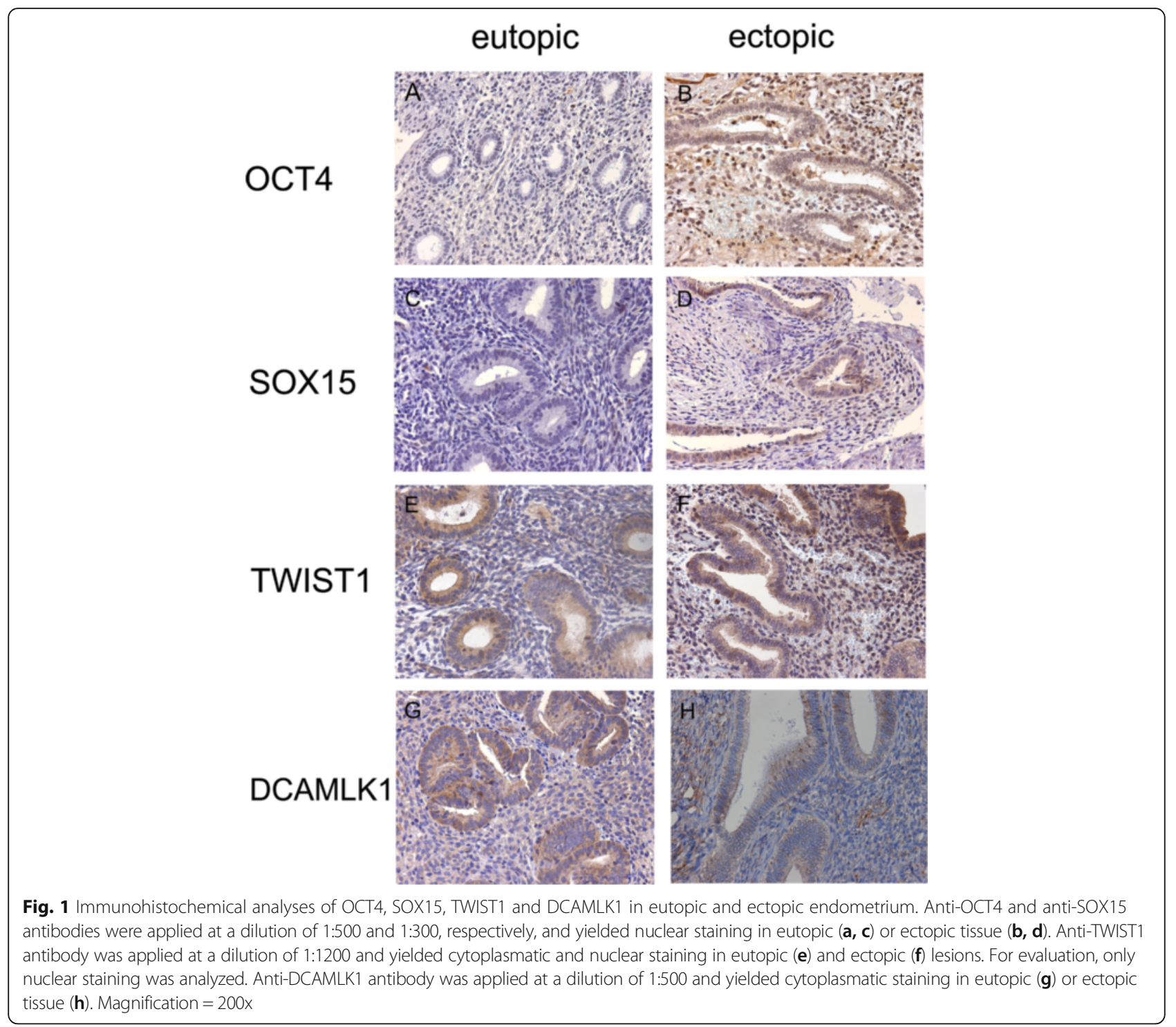

was performed in 10mM Sodium Citrate Buffer pH6. Nonspecific background staining was blocked by incubating in $0.05 \%$ fish skin in PBS. The rabbit polyclonal anti-SOX15 antibody (Abcam, ab55960, Cambridge, $\mathrm{UK}$ ) and the mouse monoclonal anti-OCT4 antibody (Abcam, ab184665, Cambridge, UK) were applied at a dilution of 1:100 and incubated over night at $4^{\circ} \mathrm{C}$. Secondary antibodies from Alexa (life technologies CA, USA, goat anti-mouse IgG: Fluor 546 (red), A-11018, goat anti-rabbit IgG, Fluor 488 (green), A11070) were diluted 1:1000 with $0.05 \%$ fish skin and incubated with $1 \mu \mathrm{g} / \mathrm{ml}$ DAPI at room temperature for one hour. The slides were mounted with Fluoromount-G (Southern Biotech 0100-01, AL, USA). Colocalization of SOX15 and OCT4 was analyzed at 630x and 945x magnification using a Zeiss LSM 700 photomicroscope and LSM software (Zeiss, Göttingen, Germany).

\section{Statistical analysis}

Data were analyzed using SPSS (17.0, IBM Corp., NY, USA). For correlation analysis, Spearman test was used. For paired statistics, the McNemar test and paired t-test were used. Unpaired Student t-test was used to compare two groups. To account for repeated measures we compared expression differences between EP endometrium and endometriotic tissue using separate tests in the paired (paired $\mathrm{t}$-test) and unpaired patient subsets (Welch $\mathrm{t}$-test). To obtain a p-value for the overall comparison between $\mathrm{EP}$ endometrium and endometriotic tissue the resulting $\mathrm{p}$ values were combined using Fisher combination test [31]. Analyses for the Fisher combination tests were performed using $\mathrm{R}$ software (R-project.org). We consider the subgroup analyzes as exploratory, and hence did not adjust for multiple testing, as recommended by Bender and Lange [32]. Statistical significance was defined as $p<0.05$. 


\section{Results}

Increased OCT4 expression in epithelium of endometriotic tissue

In a first step we investigated the OCT4 expression in EP endometrium and endometriotic tissue. Analysis of the 49 endometriosis cases with paired samples (i.e. concomitant EP endometrium and endometriotic tissue) demonstrated a significant increase of OCT4 in glandular epithelium of endometriotic tissue $(p=0.021$; paired $t$-test, Fig. 2a). No significant difference was found in the stroma (Fig. 2a). In a next step we compared the samples of the whole cohort of 110 endometriosis patients (including unpaired cases) with the 50 control cases. Similarly, epithelial OCT4 expression was significantly increased in endometriotic tissue compared to EP endometrium ( $p=0.040$, Fisher combination test, Fig. 3a) and control endometrium $(p=0.006, t$-test, Fig. 3a). The stroma showed no significant differences (Fig. 3a). Similar to the epithelial protein expression of OCT4, OCT4 mRNA expression was significantly increased in endometriotic tissue compared to EP endometrium of paired samples $(p=0.038$; paired $t$-test, Fig. $4 \mathrm{a})$. Therefore we concluded that OCT4 is overexpressed in the epithelium of endometriotic tissue.

\section{Increased SOX15 expression in epithelium of}

\section{endometriotic tissue}

Next we analyzed SOX15 expression. Similar to OCT4, SOX15 expression was significantly increased in the epithelium of endometriotic tissue compared to EP endometrium (paired samples $p<0.001$; paired $t$-test, Fig. 2b; whole cohort including unpaired samples $p<0.001$; Fisher combination test, Fig. 3b). SOX15 was also significantly increased in the epithelium of endometriotic tissue compared to control endometrium ( $p=0.029$; $t$-test, Fig. $3 \mathrm{~b})$. The analysis of stromal SOX15 expression revealed no significant difference in paired samples (Fig. 2b). In contrast to the paired samples, significant differences were found for stromal SOX15 when investigating all cases (endometriotic tissue vs EP endometrium $p=0.030$, Fisher combination test, Fig. 3b; EP endometrium vs control endometrium $p<0.001, t$-test, Fig. $3 b$; endometriotic tissue vs. control endometrium $p=0.003, t$-test, Fig. $3 b)$. Similar to the epithelial protein expression of SOX15, SOX15 mRNA expression was significantly increased in endometriotic tissue compared to EP endometrium of paired samples $(p=0.004$; paired $t$-test, Fig. 4b; $p=0.041$; McNemar, Table 2). We concluded that - similar to OCT4 - SOX15 is significantly overexpressed in the epithelium of the endometriotic tissue.

\section{Increased TWIST1 expression in epithelium of endometriotic tissue}

The analysis of TWIST1 revealed that TWIST1 expression was significantly increased in the epithelium of endometriotic tissue (paired samples $p<0.001$; paired $t$-test, Fig. 2c; all endometriosis patients including the unpaired samples $p=0.001$; Fisher combination test, Fig. 3c). The
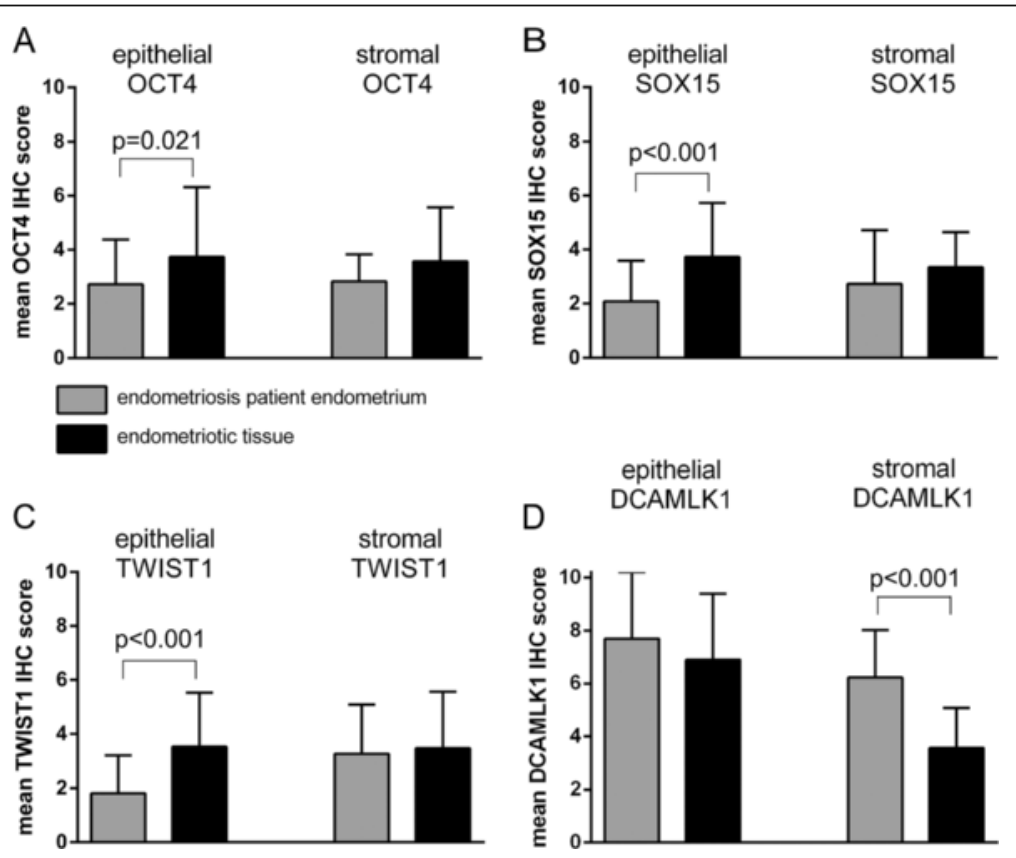

Fig. 2 Expression analyses in 49 paired cases with endometriosis. IHC was used to analyze the protein expression of OCT4 (a), SOX15 (b), TWIST1 (c) and DCAMLK1 (d). Results are expressed as mean score of the immunohistochemical staining \pm SD. Epithelial and stromal expression was analyzed separately. All p-values of paired comparisons were analysed by paired $t$-test 


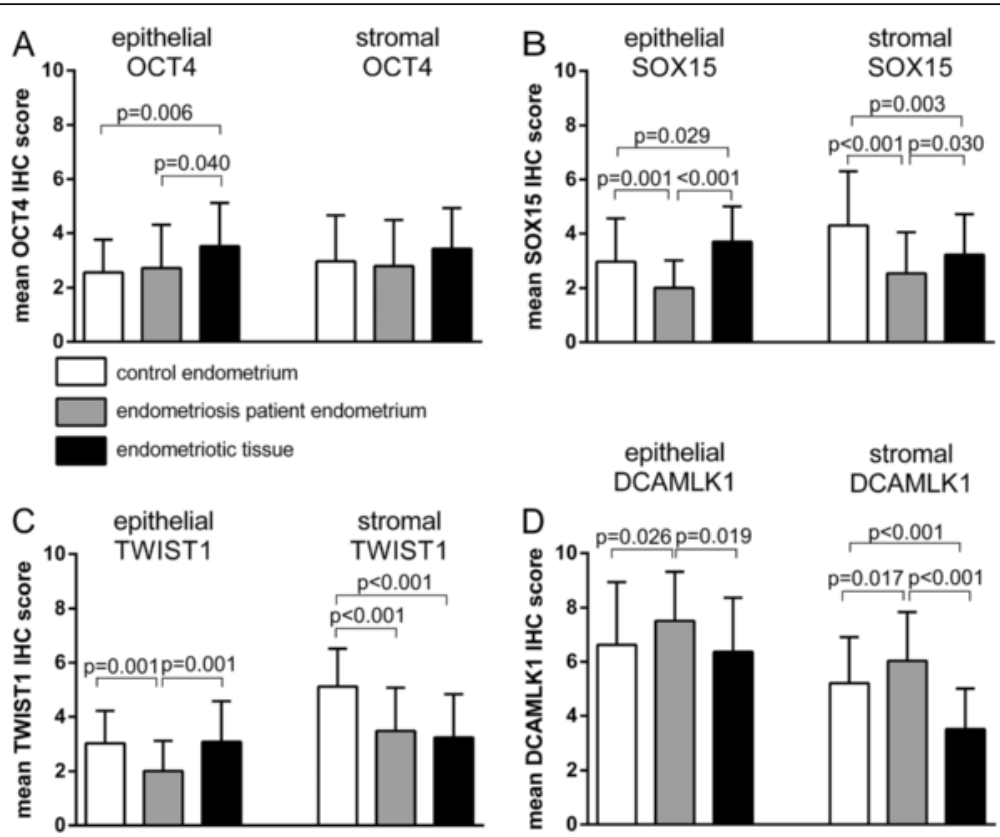

Fig. 3 Expression analyses in 50 control patients, and 110 patients with endometriosis (eutopic and ectopic). IHC was used to analyze the protein expression of OCT4 (a), SOX15 (b), TWIST1 (c) and DCAMLK1 (d). Results are expressed as mean score of the immunohistochemical staining \pm SD. Epithelial and stromal expression was analyzed separately. Comparisons with the control group were analysed by $t$-test, comparisons between EP endometrium and endometriotic tissue were performed using Fisher combination test
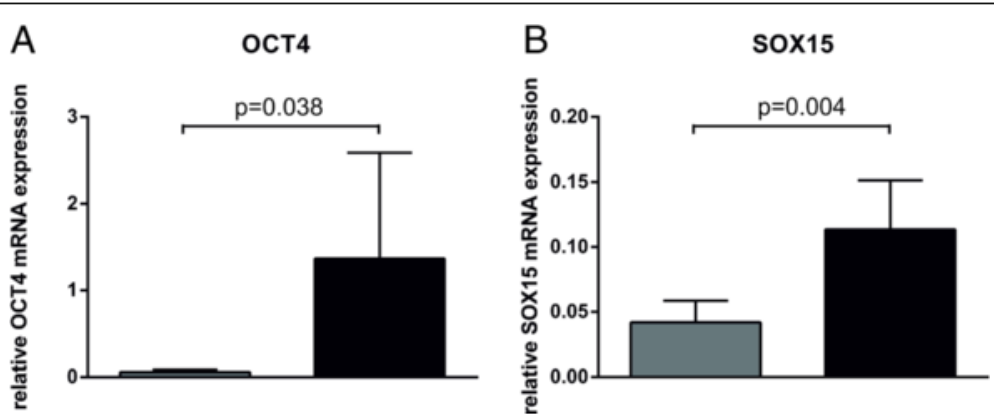

$\square$ eutopic endometrium of endometriosis patients

ectopic endometrium of endometriosis patients

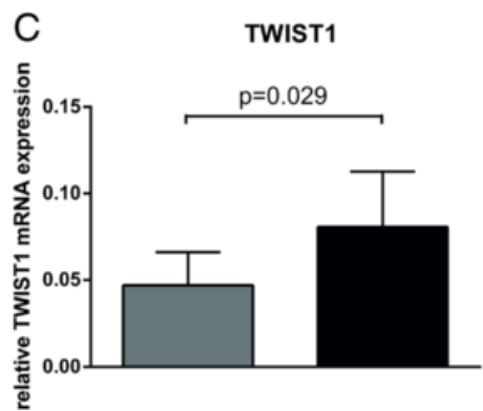

Fig. 4 Relative mRNA expression levels in 49 paired cases with endometriosis. Quantitative Real Time PCR was used to analyze the mRNA expression levels of OCT4 (a), SOX15 (b), and TWIST1 (c). Expression levels were normalized to ß-actin and GAPDH. Expression levels were shown as mean \pm SD. All $p$-values were analysed by paired $t$-test 
Table 2 SOX15 mRNA expression in EP endometrium and endometriotic tissue of the same patient

\begin{tabular}{llllllll}
\hline & \multicolumn{5}{c}{ SOX15 mRNA in endometriotic tissue } \\
\cline { 2 - 7 } & & Total & neg & pos & P-value \\
\hline SOX15 mRNA in EP & neg & 17 & 2 & $(11.8 \%)$ & 15 & $(88.2 \%)$ & 0.041 \\
endometrium & pos & 12 & 5 & $(41.7 \%)$ & 7 & (58.3\%) & \\
\hline
\end{tabular}

Numbers of patients in each of the indicated subgroups are shown. Numbers in parentheses indicate the fraction of patients (\%) in each row in ectopic endometriotic lesions negative and positive for SOX15. All p-values of subgroup comparisons were analysed by McNemar Test

analysis of stromal TWIST1 expression revealed no significant differences between endometriotic tissue and EP endometrium in paired and unpaired analysis (Fig. 2c, Fig. 3c). Further a higher stromal TWIST1 expression was found in the control endometrium compared to EP endometrium and endometriotic tissue (both $p<0.001$, Fig. 3c). A higher epithelial TWIST1 expression was found in the control endometrium compared to EP endometrium ( $p=0.001$, Fig. $3 \mathrm{c})$. Similar to the epithelial protein expression of TWIST1, TWIST1 mRNA expression was significantly increased in endometriotic tissue compared to EP endometrium of paired samples $(p=0.029$; paired $t$-test, Fig. 4c).

In conclusion, epithelial TWIST1, SOX15, and OCT4 are overexpressed in endometriotic tissue compared to EP endometrium of paired and unpaired endometriosis samples. No significant differences were found in the stroma of the paired endometriosis cases.

\section{Reduced DCAMLK1 expression in endometriotic tissue}

In the analysis of endometriosis patients, epithelial and stromal DCAMLK1 expression was found to be significantly reduced in endometriotic tissue compared to EP endometrium in paired (stromal: $p<0.001$; paired $t$-test, Fig. 2d) and unpaired samples (epithelial: $p=0.019$, stromal: $p<0.001$; Fisher combination test, Fig. $3 d$ ). In the comparison of endometriosis and control patients, epithelial and stromal DCAMLK1 expression was significantly increased in EP endometrium (epithelial: $p=$ 0.026; stromal: $p=0.017, t$-test, Fig. $3 \mathrm{~d}$ ). The mRNA levels of DCAMLK1 determined by qRT-PCR were very low in all samples and occasionally below detection level (Additional file 1). We concluded that DCAMLK1 is downregulated in ectopic endometrium. This is in contrast to the findings for OCT4, SOX15, and TWIST.

\section{OCT4, SOX15 and TWIST1 but not DCAMLK1 expressions} are positively correlated in endometriotic tissue

Next we correlated the four markers. A positive correlation was observed between epithelial OCT4 and epithelial SOX15 expression in endometriotic tissue $(p<0.001$, Spearman's rho 0.434, Table 3). Epithelial OCT4 expression also significantly correlated, although to a lower degree, with epithelial TWIST1 expression in endometriotic tissue (Spearman's rho 0.336, $p=0.002$, Table 3). A significant but very low correlation was also shown between ectopic epithelial SOX15 and TWIST1 expression $(p=0.019$, Spearman's rho 0.253, Table 3). No significant correlations were shown between DCAMLK1 and OCT4, SOX15 or TWIST1 in endometriotic tissue (Table 3). Thus we concluded that OCT4 predominately correlated with SOX15 expression.

\section{Concordant expression of OCT4 and SOX15}

We analyzed the concurrent expression of OCT4 and SOX15. 71.7\% (33/46) of endometriotic samples of the endometriosis cohort showing positive epithelial expression of OCT4 simultaneously showed positive epithelial staining for SOX15 (data not shown). In stromal cells of endometriotic tissue, $57.8 \%(26 / 45)$ of the samples with positive OCT4 staining also showed positive SOX15 expression (data not shown). There was no significant difference between SOX15 and OCT4 expression in the epithelium ( $p=0.584$, McNemar) or stroma $(p=0.643$, McNemar) analyzed in the same endometriotic tissue sample (data not shown).

Confocal immunofluorescence microscopy revealed nuclear staining of epithelial SOX15, which mostly colocalized with epithelial OCT4. Some stromal cells showed OCT4 staining without SOX15 expression (white arrows, Fig. 5). Similarly to OCT4 and SOX15 expression, TWIST1 and SOX15 mRNA were also expressed concordantly. Best concordance was observed in endometriotic tissue where $73.8 \%$ (31/42) of SOX15 mRNA positive samples were also positive for TWIST1 mRNA expression (data not shown). There was no significant difference between SOX15 and TWIST1 mRNA expression in the same endometriotic tissue sample ( $p=0.839$, McNemar, data not shown).

\section{Expression of epithelial OCT4, SOX15 and TWIST1 does not correlate with cycle phase in patients with endometriosis nor with endometriosis staging} No significant difference in OCT4, SOX15, TWIST1 or DCAMLK1 expression was observed between proliferative and secretory phase neither in EP endometrium nor in endometriotic tissue (data not shown). In contrast, in control endometrium epithelial expression of DCAMLK1 is higher in patients in proliferative cycle phase than in secretory phase $(p<0.001, t$-test, Fig. 6$)$. The expression levels of epithelial TWIST1 and OCT4 are significantly reduced in the proliferative phase of control patients ( $p=0.031$ and $p=0.006, t$-test, Fig.6). The epithelial expression of SOX15 is also reduced in proliferative phase of control patients, however at a non-significant level (Fig. 6). 
Table 3 Spearman's correlation between the epithelial expression of OCT4, SOX15, TWIST1 and DCAMLK1 in endometriotic tissue

\begin{tabular}{|c|c|c|c|c|c|}
\hline & & OCT4 & SOX15 & TWIST1 & DCAMLK1 \\
\hline \multirow[t]{3}{*}{ OCT4 } & Corr. Coefficient & 1 & 0.434 & 0.336 & 0.078 \\
\hline & Sig. (2-tailed) & & $2.63 \times 10^{-5}$ & 0.002 & 0.477 \\
\hline & $\mathrm{n}$ & 88 & 87 & 85 & 85 \\
\hline \multirow[t]{3}{*}{ SOX15 } & Corr. Coefficient & & 1 & 0.253 & 0.072 \\
\hline & Sig. (2-tailed) & & & 0.019 & 0.513 \\
\hline & $\mathrm{n}$ & & 89 & 85 & 86 \\
\hline \multirow[t]{3}{*}{ TWIST1 } & Corr. Coefficient & & & 1 & 0.14 \\
\hline & Sig. (2-tailed) & & & & 0.204 \\
\hline & $n$ & & & 86 & 84 \\
\hline \multirow[t]{3}{*}{ DCAMLK1 } & Corr. Coefficient & & & & 1 \\
\hline & Sig. (2-tailed) & & & & \\
\hline & $\mathrm{n}$ & & & & 87 \\
\hline
\end{tabular}

Expression of all factors was determined by immunohistochemistry. Correlation Coefficients and $p$-values with statistically significant $p$-values were emphasised Bold.
No significant difference in OCT4, SOX15, TWIST1 or DCAMLK1 expression was observed between the different stages of endometriosis according to the rAFS classification.

\section{Discussion}

In the present study we document the possible importance of stem cells for the development of endometriosis through the analysis of four stemness-related markers in EP endometrium, control endometrium and endometriotic tissue. Functional analyses of stem cell properties in endometriotic cells are quite limited. However, elevated expression of OCT4 and SOX2 in human endometrial cells contributed to reprogramming these cells into pluripotent stem cells (iPS cells) suggesting that OCT4 and SOX2 are stem cell factors in human endometrium [24]. SOX-OCT transcription factor combinations correlate with the efficiency in producing iPS
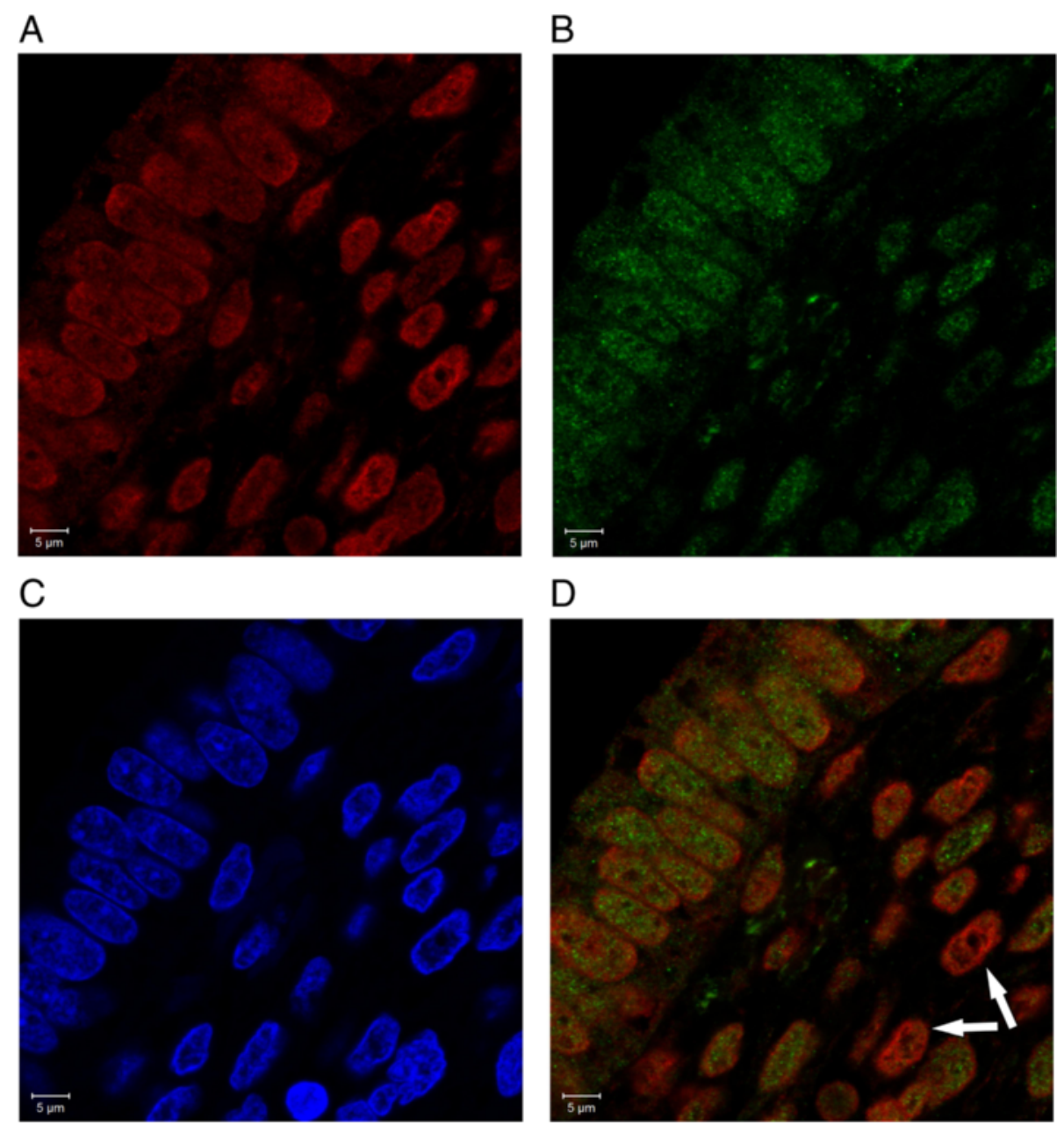

Fig. 5 Colocalization of OCT4 and SOX15 in human endometriotic tissue. Paraffin sections of human endometrium were processed for immunofluorescence microscopy. OCT4 (a), SOX15 (b), and DAPI (c) stainings are shown. OCT4/SOX15 merged picture (d) denotes colocalization in all epithelial cells whereas few stromal cells were only positive for OCT4 (white arrows) 


\section{Epithelial expression in controls}

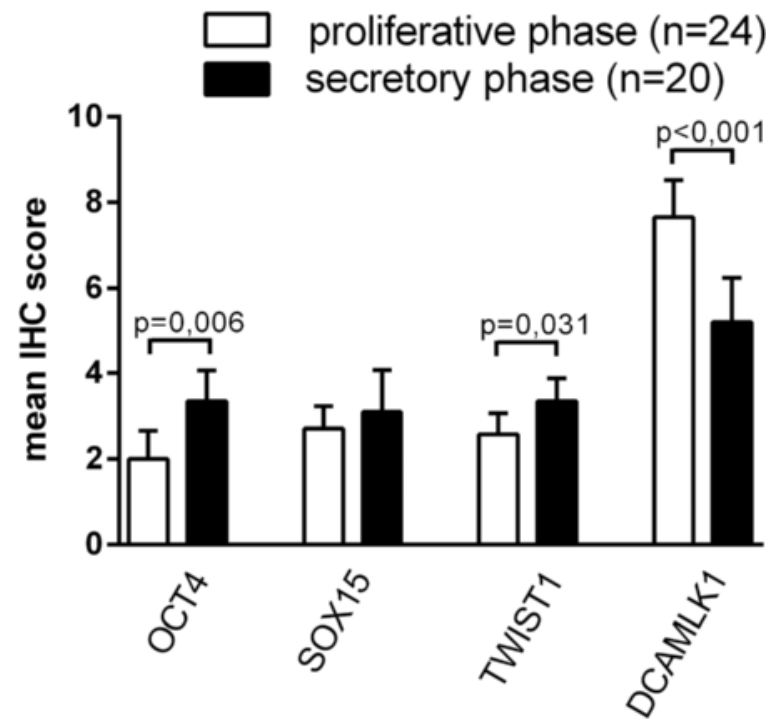

Fig. 6 Expression levels of epithelial OCT4, SOX15, TWIST1 and DCAMLK1 in proliferative and secretory phase of the menstrual cycle in control patients. Results are expressed as mean scores of the immunohistochemical stainings \pm SD. All p-values of subgroup comparisons were analysed by t-test

cells. Thus we also analyzed a potential correlation and coexpression of OCT4 and SOX15, since SOX15 protein expression has never been studied in endometriosis [28]. We studied OCT4 and SOX15 expression in 110 cases and confirmed a positive correlation and overexpression of both factors in the epithelium of endometriotic tissue. Our findings are in line with previous studies with smaller sample sizes reporting an increased expression of stemness-related markers in endometriosis $[25,26]$. SOX2 expression has been shown to be significantly increased in endometriosis compared to secretory endometrium of patients without endometriosis [33]. Musashi-1, an epithelial progenitor cell marker regulating self-renewal pathways, was also found to be overexpressed in endometriotic tissue compared to eutopic endometrium of controls [34]. SALL4, which is a transcriptional regulator of OCT4 and a marker for pluripotency, has been shown to be expressed in endometriotic tissue but not in EP endometrium [26]. Interestingly, SOX15 and TWIST1 expression was lower in EP endometrium compared to the control endometrium. These factors may be upregulated in endometrial cells after dissemination to promote cell survival and invasion predominantly outside the uterine cavity in order to form endometriotic lesions.

The enhanced expression of stemness-related markers in endometriotic tissue may foster self-renewal and increase cell survival. Analysis of eutopic endometrial cells collected from the menstrual blood of patients with and without endometriosis revealed that endometriosis patients had higher mRNA expression of OCT4 and SOX2 than normal controls [35]. Furthermore, women with endometriosis have larger volumes of retrograde menstrual flow than women without endometriosis and baboons whose cervices had been ligated showed a significant increase in development of endometriosis [36, 37]. Thus, our findings may support the hypothesis that cells with stem cell capacity, disseminated into the peritoneal cavity via retrograde menstrual efflux, may accumulate and contribute to the establishment of ectopic endometriotic lesions [7-11]. However, another proposal concerning the histologic origin of endometriosis is that circulating blood cells originating from bone marrow can differentiate into endometriotic tissue at various sites [38]. According to this, a small number of the accumulated stem cells shown in the endometrium of endometriosis patients may also derive from bone marrow. Moreover, it is unclear if increased stem cell number or even abnormal stem cell properties facilitate the establishment of ectopic endometrial implants in patients developing endometriosis.

We and others found an overexpression of TWIST1 in ectopic endometrium [39, 40]. TWIST1 is a marker for epithelial to mesenchymal transition (EMT), and increases the migratory activity of endometrial cells [39]. Recent evidence suggest that cells undergoing EMT acquire stem cell-like properties and the mesenchymal status seems to be a condition to regain pluripotency [41]. Thus, TWIST1 could be another potential stemness-related marker in human endometrium.

DCAMLK1, a microtubule-associated kinase, is a putative stem cell marker in pancreas and intestine, which regulates TWIST1, MYC, KRAS and other factors. DCAMLK1 was identified as a novel pancreatic cancer stem cell marker [42]. Interestingly, DCAMLK1 has the capacity to distinguish between normal and tumor stem cells at least in the intestine [43]. So far, no study has analyzed the expression of DCAMLK1 in endometriosis. Surprisingly, we found significant results for DCAMLK1 with an ectopic expression pattern inverse to OCT4. Stromal and epithelial DCAMLK1 expression was significantly higher in EP endometrium than in endometriotic tissue. The expression of stromal and epithelial DCAMLK1 was significantly increased in EP endometrium compared to control endometrium. This finding suggests that in endometriosis patients DCAMLK1 is upregulated in cells of the uterine cavity before dissemination into the peritoneal cavity. The upregulation of DCAMLK1 in EP endometrium may be due to enhanced posttranslational stabilization of the protein as the mRNA levels of DCAMLK1 were very 
low. Although, it cannot be excluded that the diverse expression pattern of DCAMLK1 is unrelated to stem cell properties, our findings may indicate that eutopic upregulation of DCAMLK1 may be of importance for endometriotic cell survival before the establishment of endometriotic lesions.

The correlation of stem cell factors with menstrual cycle is controversial. It has been reported that the number of clonogenic epithelial and stromal cells did not vary between proliferative and secretory endometrium in women without endometriosis [6]. Furthermore, most studies did not find a correlation between the expression of stemness-related factors and the phase of the menstrual cycle $[19,20,26,34]$. This is in concordance with our findings showing no correlation between the epithelial or stromal expression of the stemness-related markers DCAMLK1, OCT4, SOX15 and TWIST1 and the cycle phase of endometriosis patients, neither in EP endometrium nor in endometriotic lesions. In contrast, few studies found an increased expression of stemness-related markers, such as SOX2 or Musashi-1, in proliferative phase [33, 34]. However, in patients without endometriosis, we found significantly higher epithelial expression of DCAMLK1 in the proliferative cycle phase than in the secretory phase whereas the expression levels of epithelial OCT4, SOX15 and TWIST1 are rather reduced in the proliferative phase of patients without endometriosis. Therefore, it has to be pointed out that some differences of the expression levels of stemness-related factors between endometrium of controls and patients may be hormonally related. Generally, an upregulation of stemness-related factors in normal endometrium possibly prepares the tissue for renewal by accumulation of pluripotent cells in the end of the menstrual cycle.

Lastly, it must be mentioned that even for the established stem cell marker OCT4 a formal proof of stem cell property is still lacking in endometriotic cells. Thus in endometriotic cells colony forming assays after transduction or knockdown of these stemness-related factors would be of great interest.

\section{Conclusion}

In conclusion, the results reported herein suggest that in endometriosis SOX15 and TWIST1 may be stemnessrelated markers as their expression correlates with OCT4 expression. The epithelial expression of OCT4, SOX15 and TWIST1 is increased in endometriotic tissue compared to EP endometrium in paired and unpaired analysis supporting the hypothesis that upregulation of stem cellrelated markers contribute to the establishment of ectopic endometriotic lesions.

\section{Additional file}

Additional file 1: Quantitative Real Time PCR was used to analyze the mRNA expression levels of DCAMLK1. Representative qRT-PCR results ( Ct values) for DCAMLK1 and control genes (ACTB and GAPDH) are shown. (DOCX 216 kb)

\section{Abbreviations}

bcl-2: B-cell cll/lymphoma 2; CD34: Hematopoietic progenitor cell antigen CD34; c-kit: Kit, V-kit Hardy-Zuckerman 4 feline sarcoma viral oncogene homolog, cd117; DCAMLK1: Doublecortin- and calmodulin kinase-like 1; EM: endometriosis; EMT: epithelial to mesenchymal transition; iPS

cells: induced pluripotent stem cells; KRAS: V-KI-RAS2 Kirsten rat sarcoma viral oncogene homolog; Musashi-1: Musashi, drosophila, homolog of, 1; MYC: VMYC Avian myelocytomatosis viral oncogene homolog; na: status not available; OCT4: Octamer-binding transcription factor 4; qRT-PCR: quantitative Real-Time Polymerase Chain Reaction; rAFS: revised American fertility society; SALL4: Sal-Like 4; SOX15: Sry-box 15; SOX2: Sry-Box 2; TWIST1: Drosophila homolog of 1

\section{Acknowledgements}

Sincere thanks to all doctors and nurses, who assisted with tissue collection. Special thanks go to Manuela Gstöttner and Philipp Pappenscheller from the Viennese Endometriosezentrum. Thanks to Christoph Hauser for technical assistance and Florian Klinglmueller for statistical support.

\section{Funding}

This study was funded by the Ingrid Flick Foundation.

\section{Availability of data and material}

The complete dataset supporting the conclusions of this article is kept in an anonymous file at the archive at the Department of Gynecology and Obstetrics, Medical University of Vienna, according to regulations stated by the institutional review board, in order to protect the participants' identity. However, detailed tables were included in the manuscript.

\section{Authors' contributions}

$\mathrm{KP}, \mathrm{BS}$ and $\mathrm{HH}$ made substantial contributions to conception, design, acquisition of data, analysis and interpretation of data. KP and BS were involved in drafting the manuscript and revising it critically for intellectual content. BP assisted in concept development and manuscript preparation, recruitment of patients, acquisition, analysis and interpretation of data. SB made contributions to acquisition of data, performed statistical analysis, and reviewed the manuscript. TR, NN and EM made contributions to acquisition of data, performed statistical analysis and reviewed the manuscript. RW, HH, GY and LK made substantial contributions in recruitment of patients, provided clinical information and assisted in manuscript preparation. $\mathrm{HH}$ made substantial contributions to concept development, study design and revised it critically for important intellectual content. All authors read and approved the final manuscript.

\section{Author's information}

Not applicable.

\section{Competing interests}

The authors declare that they have no competing interests.

\section{Consent for publication}

All participants provided written informed consent.

\section{Ethics approval and consent to participate}

The study was approved by the institutional review board (545/2010) of the Medical University of Vienna.

\section{Author details}

'Department of Obstetrics and Gynecology, Medical University of Vienna, Waehringer Guertel 18-20, 1090 Vienna, Austria. ²Department of Pathology, Medical University of Vienna, Waehringer Guertel 18-20, 1090 Vienna, Austria. ${ }^{3}$ Fetal Medicine Research Institute, King's College Hospital, 16-20 Windsor Walk, Denmark Hill, SE58BB London, UK. 
Received: 15 July 2016 Accepted: 7 November 2016 Published online: 24 November 2016

\section{References}

1. Giudice LC, Kao LC. Endometriosis. Lancet. 2004;364:1789-99.

2. Sampson JA. Metastatic or embolic endometriosis, due to the menstrual dissemination of endometrial tissue into the venous circulation. Am J Pathol. 1927:3:93-110. 143.

3. Maruyama T, Yoshimura Y. Molecular and cellular mechanisms for differentiation and regeneration of the uterine endometrium. Endocr J. 2008:55:795-810.

4. Chan RW, Schwab KE, Gargett CE. Clonogenicity of human endometrial epithelial and stromal cells. Biol Reprod. 2004;70:1738-50.

5. Gargett CE. Stem cells in gynaecology. Aust N Z J Obstet Gynaecol. 2004:44:380-6.

6. Schwab KE, Chan RW, Gargett CE. Putative stem cell activity of human endometrial epithelial and stromal cells during the menstrual cycle. Fertil Steril. 2005;84 Suppl 2:1124-30.

7. Figueira PG, Abrao MS, Krikun G, Taylor HS. Stem cells in endometrium and their role in the pathogenesis of endometriosis. Ann N Y Acad Sci. 2011;1221:10-7.

8. Sasson IE, Taylor HS. Stem cells and the pathogenesis of endometriosis. Ann N Y Acad Sci. 2008;1127:106-15.

9. Gargett BE, Chan RW. Endometrial stem/progenitor cells and proliferative disorders of the endometrium. Minerva Ginecol. 2006;58:511-26.

10. Kim CM, Oh YJ, Cho SH, Chung DJ, Hwang JY, Park KH, Cho DJ, Choi YM, Lee BS. Increased telomerase activity and human telomerase reverse transcriptase mRNA expression in the endometrium of patients with endometriosis. Hum Reprod. 2007:22:843-9.

11. Starzinski-Powitz A, Zeitvogel A, Schreiner A, Baumann R. [Endometriosis-a stem cell disease?]. Zentralbl Gynakol. 2003;125:235-8.

12. Jimbo H, Hitomi Y, Yoshikawa H, Yano T, Momoeda M, Sakamoto A, Tsutsumi O, Taketani Y, Esumi H. Evidence for monoclonal expansion of epithelial cells in ovarian endometrial cysts. Am J Pathol. 1997;150:1173-8.

13. Meng X, Ichim TE, Zhong J, Rogers A, Yin Z, Jackson J, Wang H, Ge W Bogin V, Chan KW, et al. Endometrial regenerative cells: a novel stem cell population. J Transl Med. 2007:5:57

14. Tamura M, Fukaya T, Murakami T, Uehara S, Yajima A. Analysis of clonality in human endometriotic cysts based on evaluation of $X$ chromosome inactivation in archival formalin-fixed, paraffin-embedded tissue. Lab Investig. 1998;78:213-8.

15. Tanaka M, Kyo S, Kanaya T, Yatabe N, Nakamura M, Maida Y, Okabe M, Inoue M. Evidence of the monoclonal composition of human endometrial epithelial glands and mosaic pattern of clonal distribution in luminal epithelium. Am J Pathol. 2003;163:295-301.

16. Tanaka T, Nakajima S, Umesaki N. Cellular heterogeneity in long-term surviving cells isolated from eutopic endometrial, ovarian endometrioma and adenomyosis tissues. Oncol Rep. 2003:10:1155-60.

17. Wu Y, Basir Z, Kajdacsy-Balla A, Strawn E, Macias V, Montgomery K, Guo SW. Resolution of clonal origins for endometriotic lesions using laser capture microdissection and the human androgen receptor (HUMARA) assay. Fertil Steril. 2003;79 Suppl 1:710-7.

18. Cho NH, Park YK, Kim YT, Yang H, Kim SK. Lifetime expression of stem cell markers in the uterine endometrium. Fertil Steril. 2004;81:403-7.

19. Matthai C, Horvat R, Noe M, Nagele F, Radjabi A, van Trotsenburg M, Huber J, Kolbus A. Oct-4 expression in human endometrium. Mol Hum Reprod. 2006;12:7-10.

20. Bentz EK, Kenning M, Schneeberger C, Kolbus A, Huber JC, Hefler LA, Tempfer CB. OCT-4 expression in follicular and luteal phase endometrium: a pilot study. Reproductive biology and endocrinology : RB\&E. 2010;8:38.

21. Kato K, Yoshimoto M, Adachi S, Yamayoshi A, Arima T, Asanoma K, Kyo S, Nakahata T, Wake N. Characterization of side-population cells in human normal endometrium. Hum Reprod. 2007;22:1214-23.

22. Lynch L, Golden-Mason L, Eogan M, O'Herlihy C, O'Farrelly C. Cells with haematopoietic stem cell phenotype in adult human endometrium: relevance to infertility? Hum Reprod. 2007:22:919-26.

23. Tai MH, Chang CC, Kiupel M, Webster JD, Olson LK, Trosko JE. Oct4 expression in adult human stem cells: evidence in support of the stem cell theory of carcinogenesis. Carcinogenesis. 2005;26:495-502.

24. Park JH, Daheron L, Kantarci S, Lee BS, Teixeira JM. Human endometrial cells express elevated levels of pluripotent factors and are more amenable to reprogramming into induced pluripotent stem cells. Endocrinology 2011:152:1080-9.

25. Pacchiarotti A, Caserta D, Sbracia M, Moscarini M. Expression of oct-4 and c-kit antigens in endometriosis. Fertil Steril. 2011:95:1171-3.

26. Forte A, Schettino MT, Finicelli M, Cipollaro M, Colacurci N, Cobellis L, Galderisi U. Expression pattern of stemness-related genes in human endometrial and endometriotic tissues. Mol Med. 2009;15:392-401.

27. Zeineddine D, Hammoud AA, Mortada M, Boeuf H. The Oct4 protein: more than a magic stemness marker. American J Stem Cells. 2014;3:74-82.

28. Ng CK, Li NX, Chee S, Prabhakar S, Kolatkar PR, Jauch R. Deciphering the Sox-Oct partner code by quantitative cooperativity measurements. Nucleic Acids Res. 2012;40:4933-41.

29. Revised American Society for Reproductive Medicine classification of endometriosis: 1996. Fertility and sterility 1997, 67:817-821.

30. Proestling K, Hebar A, Pruckner N, Marton E, Vinatzer U, Schreiber M. The Pro allele of the p53 codon 72 polymorphism is associated with decreased intratumoral expression of BAX and p21, and increased breast cancer risk. PLoS One. 2012;7, e47325.

31. Fisher RA. Statistical Methods for Research Workers. Oliver and Boyd. Edingburgh: Oliver and Boyd; 1925.

32. Bender R, Lange S. Adjusting for multiple testing-when and how? J Clin Epidemiol. 2001:54:343-9.

33. Gotte M, Wolf M, Staebler A, Buchweitz O, Kiesel L, Schuring AN. Aberrant expression of the pluripotency marker SOX-2 in endometriosis. Fertil Steril. 2011;95:338-41.

34. Gotte M, Wolf M, Staebler A, Buchweitz O, Kelsch R, Schuring AN, Kiesel L. Increased expression of the adult stem cell marker Musashi- 1 in endometriosis and endometrial carcinoma. J Pathol. 2008:215:317-29.

35. Hwang JH, Oh JJ, Wang T, Jin YC, Lee JS, Choi JR, Lee KS, Joo JK, Lee HG. Identification of biomarkers for endometriosis in eutopic endometrial cells from patients with endometriosis using a proteomics approach. Mol Med Rep. 2013;8:183-8.

36. Halme J, Hammond MG, Hulka JF, Raj SG, Talbert LM. Retrograde menstruation in healthy women and in patients with endometriosis. Obstet Gynecol. 1984;64:151-4.

37. D'Hooghe TM. Clinical relevance of the baboon as a model for the study of endometriosis. Fertil Steril. 1997:68:613-25.

38. Du H, Taylor HS. Contribution of bone marrow-derived stem cells to endometrium and endometriosis. Stem Cells. 2007;25:2082-6.

39. Chang JH, Au HK, Lee WC, Chi CC, Ling TY, Wang LM, Kao SH, Huang YH, Tzeng CR. Expression of the pluripotent transcription factor OCT4 promotes cell migration in endometriosis. Fertil Steril. 2013;99:1332-9. e1335.

40. Proestling K, Birner P, Gamperl S, Nirtl N, Marton E, Yerlikaya G, Wenzl R, Streubel B, Husslein H. Enhanced epithelial to mesenchymal transition (EMT) and upregulated MYC in ectopic lesions contribute independently to endometriosis. Reprod Biol Endocrinol. 2015;13:75.

41. Thiery JP, Acloque H, Huang RY, Nieto MA. Epithelial-mesenchymal transitions in development and disease. Cell. 2009:139:871-90.

42. Sureban SM, May R, Lightfoot SA, Hoskins AB, Lerner M, Brackett DJ, Postier RG, Ramanujam R, Mohammed A, Rao CV, et al. DCAMKL-1 regulates epithelial-mesenchymal transition in human pancreatic cells through a miR200a-dependent mechanism. Cancer Res. 2011;71:2328-38.

43. Nakanishi Y, Seno H, Fukuoka A, Ueo T, Yamaga Y, Maruno T, Nakanishi N, Kanda K, Komekado H, Kawada M, et al. Dclk1 distinguishes between tumor and normal stem cells in the intestine. Nat Genet. 2013:45:98-103.

\section{Submit your next manuscript to BioMed Central and we will help you at every step:}

- We accept pre-submission inquiries

- Our selector tool helps you to find the most relevant journal

- We provide round the clock customer support

- Convenient online submission

- Thorough peer review

- Inclusion in PubMed and all major indexing services

- Maximum visibility for your research

Submit your manuscript at www.biomedcentral.com/submit 